\title{
NARRATIVAS DIGITAIS DE PROFESSORES: UMA REVISÃO SISTEMÁTICA EM TESES E DISSERTAÇÕES BRASILEIRAS
}

\author{
Marcus Alexandre de Pádua Cavalcanti Bastos \\ Doutor em Educação em Ciências e Saúde pela Universidade Federal do Rio de Janeiro - UFRJ \\ Rio de Janeiro, RJ, Brasil \\ marcus_nathan120@hotmail.com
}

Eliane Cristina Tenório Cavalcanti

Doutoranda em Educação em Ciências e Saúde pela Universidade Federal do Rio de Janeiro - UFRJ

- Rio de Janeiro, RJ, Brasil

eliane.cavalcanti@hotmail.com.br

Marcelo de Jesus Pereira

Mestrando em Educação em Ciências -Universidade do Grande Rio, UNIGRANRIO

Rio de Janeiro, RJ, Brasil marcelo.pereira@transluxtransportes.com.br

Shirley Araújo Cabral
Mestre em Desenvolvimento Local - Centro Universitário Augusto Motta - UNISUAM
Rio de Janeiro, RJ, Brasil
shirleyacabral@gmail.com.br
Bacharelando em Farmácia Universidade Estácio de Sá - UNESA
Rio de Janeiro, RJ, Brasil
nathan-psytrance@hotmail.com

RESUMO

Este trabalho teve como objetivo realizar uma revisão sistemática de teses e dissertações que tratam de narrativas digitais de professores atuantes em escolas de Educação Básica e Educação Superior no país. Para tanto, buscou-se analisar dissertações e teses existentes no Portal de Periódicos da Capes do período de 2016 a 2018 sobre essa temática. Trata-se de uma a pesquisa exploratória e descritiva sendo que os seus dados e sua análise foram explorados de forma quantitativa e qualitativa. O material da investigação foi composto por 07 trabalhos que atenderam aos critérios de inclusão e exclusão previamente definidos. A análise permitiu observar que o tema foi pouco explorado no cenário brasileiro até 2017. Os resultados apontam que a partir de 2018 houve uma mudança significativa no que se refere ao aumento de pesquisas sobre as narrativas digitais de professores. Entre as áreas mais exploradas, o destaque é para a Educação, em seguida, a seguir estão os trabalhos na área de Ensino de Ciências e Cognição e Linguagem.

Palavras-chave: Narrativa digital. Professores. Portal da Capes 


\title{
DIGITAL NARRATIVES OF TEACHERS: A SYSTEMATIC REVIEW IN BRAZILIAN THESES AND DISSERTATIONS
}

\begin{abstract}
This work aimed to carry out a systematic review of theses and dissertations that deal with digital narratives of teachers working in schools of Basic Education and Higher Education in the country. To this end, we sought to analyze dissertations and theses existing on the Capes Journal Portal from the period 2016 to 2018 on this theme. It is an exploratory and descriptive research and its data and analysis were explored in a quantitative and qualitative way. The investigation material was composed of 07 works that met the inclusion and exclusion criteria previously defined. The analysis allowed to observe that the theme was little explored in the Brazilian scenario until 2017. The results show that, from 2018 on, there was a significant change regarding the increase in research on teachers' digital narratives. Among the most explored areas, the highlight is Education, followed by work in the area of Science and Cognition and Language Teaching.
\end{abstract}

Keywords: Digital narrative. Teachers. Capes Journals Portal 
NARRATIVAS DIGITAIS DE PROFESSORES: UMA REVISÃO SISTEMÁTICA EM TESES E DISSERTAÇÕES BRASILEIRAS
Marcus Alexandre de Pádua Cavalcanti Bastos Eliane Cristina Tenório Cavalcanti Marcelo de Jesus Pereira Shirley Araújo Cabral

Nathan da Costa Cavalcanti Bastos

\title{
1 INTRODUÇÃO
}

As narrativas fazem parte da história da humanidade, elas trazem consigo a possibilidade de contar e recontar a história, não permitindo que esta história caia no esquecimento. Narrar constitui-se, assim, como um instrumento que permite o acesso a certo tipo de conhecimento, um processo fundamental de desenvolvimento cognitivo e de percepção do real que possibilita a compreensão da representação que o sujeito faz da realidade na qual está inserido (BARTHES, 2013).

De acordo com Bruner (2007) a narrativa é um modo de pensamento, pois ela se apresenta como princípio organizador da experiência humana no mundo social, do seu conhecimento sobre ele e das trocas que ele mantém. O autor considera que a narrativa é uma importante ferramenta para a construção do significado em nossa cultura.

Recentemente, a pesquisa em educação vem adotando as narrativas de professores como um instrumento de investigação da prática docente. A narrativa é uma das formas mais comuns do professor expressar um tipo de saber (VAZ, MENDES, MAUÉS, 2001).

As pesquisas sobre narrativas buscam produzir conhecimentos que não estão ao alcance dos métodos tradicionais de investigação científica, como os significados que as pessoas dão às suas vivências, às situações e às ações de suas vidas, inseridas no grupo e na coletividade (POLKINGHORNE, 2007).

Bruner (2002, p. 46) define a narrativa como

\begin{abstract}
Uma sequência singular de eventos, estados mentais, ocorrências envolvendo seres humanos como personagens ou atores situados em determinado contexto, no tempo dos acontecimentos, permitindo avançar ou retroceder no tempo, de acordo com as intenções em processos de construção de conhecimentos e negociação de significados, que englobam as relações entre mente e cultura.
\end{abstract}

De acordo com Almeida e Valente (2012, p. 59), "as narrativas, que eram tradicionalmente orais ou escritas, podem ser agora produzidas com uma combinação de mídias, o que pode contribuir para que esta atividade seja muito mais rica e sofisticada". Em consonância com Almeida e Valente (2012), Rodrigues e Gonçalves (2014, p. 216) destacam que essas narrativas "podem ser produzidas por meio das tecnologias digitais de informação 
NARRATIVAS DIGITAIS DE PROFESSORES: UMA REVISÃO SISTEMÁTICA EM TESES E DISSERTAÇÕES BRASILEIRAS
Marcus Alexandre de Pádua Cavalcanti Bastos Eliane Cristina Tenório Cavalcanti Marcelo de Jesus Pereira Shirley Araújo Cabral

Nathan da Costa Cavalcanti Bastos

e comunicação (TDICs) e numa textualidade eletrônica que é multimidiática, o sujeito pode lançar mão de uma diversidade ainda maior de recursos para se expressar".

As narrativas têm ganhado novos significados e usos com o advento das tecnologias digitais de informação e comunicação (PALÁCIO; STRUCHINER, 2017). A habilidade da narração em tempos das TDICs vem sendo incorporada na educação (BELL, 2014). Elas são produzidas com uma combinação de mídias (ALMEIDA; VALENTE, 2012), cuja construção constitui-se "num processo de produção textual que assume o caráter contemporâneo dos recursos audiovisuais e tecnológicos capazes de modernizar 'o contar histórias', tornando-se uma ferramenta pedagógica eficiente e motivadora" (CARVALHO, 2008, p. 87), Assim, os professores passaram a ter novos suportes e possibilidades de formato digital (LISBÔA E COUTINHO, 2012):

O presente estudo tem como objetivo Identificar e analisar pesquisas realizadas em teses e dissertações que tratam de narrativas digitais de professores na Educação Básica e Superior de todo o país. Partimos do pressuposto de que o uso das narrativas digitais pelos professores potencializa a aprendizagem e influência no processo educativo.

\section{AS NARRATIVAS DIGITAIS}

As narrativas digitais emergem como mais uma possibilidade de investigação, pois esse tema agrega vários elementos além daqueles associados à oralidade e à escrita em sua forma tradicional. Almeida e Valente (2012, p. 59), apontam que "as narrativas, que eram tradicionalmente orais ou escritas, podem ser agora produzidas com uma combinação de mídias, o que pode contribuir para que esta atividade seja muito mais rica e sofisticada".

Associada aos recursos digitais, a narrativa digital vem sendo utilizada e estudada sob diversas nomenclaturas e com diferentes abordagens: digital storytelling (ROBY, 2010; CLARKE; ADAM, 2012; ROBIN; MCNEIL, 2012), narrativa multimídia (MAGALHÃES, 2010; ARAÚJO, 2010), digital stories (XIN, 2014) e digital narratives (LOVELL; BAKER, 2009).

Delas fazem parte imagens, animações, sons, links e uma série de outros recursos que compõem esse tipo de narrativa. Miller (2014) aponta que elas

Possuem caracteres possíveis apenas em meios digitais: principalmente os que podem ser controlados por computadores; Interativos: o usuário pode controlar ou 
NARRATIVAS DIGITAIS DE PROFESSORES: UMA REVISÃO SISTEMÁTICA EM TESES E DISSERTAÇÕES BRASILEIRAS
Marcus Alexandre de Pádua Cavalcanti Bastos Eliane Cristina Tenório Cavalcanti Marcelo de Jesus Pereira Shirley Araújo Cabral

Nathan da Costa Cavalcanti Bastos

impactar aspectos da história; Não lineares: os fatos narrados não ocorrem em uma ordem fixa; Profundamente imersivos: atrativos da história e do meio que conduzem e mantém o usuário dentro do enredo; Participativos: possibilidade de o usuário participar da história; Navegáveis: os usuários fazem seu próprio caminho através da história ou através do ambiente virtual (MILLER, 2014, p. 14).

Almeida e Valente (2012, p. 63) afirmam que elas "podem ser utilizadas tanto para investigar o conhecimento que as pessoas expressam quanto para auxiliar processos de construção de conhecimento". Na área da educação, elas auxiliam nos processos de produção de conhecimento (ALMEIDA E VALENTE 2012, 2014).

De acordo com Rodrigues (2017) as investigações no cenário brasileiro sobre esse tema ainda são bastante incipientes e escassas. Almeida e Valente $(2012,2014)$ ressaltam a importância das narrativas digitais na educação, uma vez que elas "podem ser produzidas com uma combinação de mídias, o que pode contribuir para que esta atividade seja muito rica e sofisticada" (ALMEIDA E VALENTE, 2012, p. 59). Os autores destacam ainda que o trabalho com as narrativas digitais é uma forma de explorar o potencial das tecnologias digitais de informação e comunicação (TDIC) em distintas áreas do conhecimento (ALMEIDA E VALENTE, 2012, p. 58). Eles ainda acrescentam que "na narrativa digital, produzida por meio das TDIC, o sujeito pode lançar mão de uma diversidade ainda maior de recursos para se expressar" (ALMEIDA E VALENTE, 2014, p. 216).

\section{PROCEDIMENTOS METODOLÓGICOS}

O método utilizado foi a Revisão Sistemática da Literatura que segundo Freire (2013, p.33) "absorve os resultados dos estudos de outros pesquisadores, tratando seus resultados como estudos secundários. Esse método foi escolhido para que se possa fazer uma síntese do conhecimento já adquirido sobre o tema e assim possibilitar caminhos para identificar possíveis avanços ao se comparar e estatisticamente relacionar os resultados de diferentes pesquisas que levem a novos resultados (VELLUZZI, 1998)

A pesquisa foi elaborada a partir da necessidade de identificar estudos sobre a utilização de Narrativas Digitais de professores atuantes em escolas de Educação Básica e Educação Superior de todo o país. A coleta dos artigos foi realizada no Banco de Teses e Dissertações da CAPES. A busca nessa base se justifica pelo fato de que ela atende às 
NARRATIVAS DIGITAIS DE PROFESSORES: UMA REVISÃO SISTEMÁTICA EM TESES E DISSERTAÇÕES BRASILEIRAS
Marcus Alexandre de Pádua Cavalcanti Bastos Eliane Cristina Tenório Cavalcanti Marcelo de Jesus Pereira Shirley Araújo Cabral

Nathan da Costa Cavalcanti Bastos

demandas dos setores acadêmico, produtivo e governamental, possibilitando a promoção do aumento da produção científica nacional e da inserção científica brasileira no exterior.

Para a realização desta revisão, foram utilizados os seguintes procedimentos: elaboração da questão de pesquisa, levantamento do material bibliográfico analisado que constituiu em teses e dissertações, cujos dados coletados foram: título, autor, ano, instituição, tipo de trabalho e programa. Estabelecemos os critérios para inclusão e exclusão de estudos, definição das informações a serem extraídas dos estudos selecionados, avaliação dos estudos incluídos na revisão, interpretação dos resultados e síntese do conhecimento.

Definiram-se como critérios de inclusão os trabalhos que abordem narrativas digitais de professores atuantes em escolas de toda a Educação Básica e em Instituições de Ensino Superior (IES). Como critério de exclusão os trabalhos que não envolvam narrativas digitais de professores. O lapso temporal abarcado foi de 2016 a 2018.

A questão que orientou esse trabalho foi a seguinte: Como as narrativas digitais vêm sendo utilizadas por professores do ensino básico e superior no país?

Condizente com essa indagação ficaram definidos para as buscas os descritores "narrativas digitais", realizou-se a busca 1, a partir da qual advieram 85 trabalhos. Logo após esta, a fim de especificar e afunilar, ainda mais, a pesquisa, lançou-se mão da equação "narrativa digital" and "professor" (BUSCA 2), por meio da qual derivaram 72 obras.

Todavia, como o quantitativo de trabalho resultante da busca 2 foi grande (72 trabalhos), optou-se por continuar a busca, desta vez, tentando com as mesmas palavraschave, mas em ordem inversa, isto é, "professor" AND "narrativa digital", resultando 7 estudos.

Após a aplicação dos critérios e o refinamento da busca a partir da leitura dos artigos pré-selecionados, a amostra restringiu-se a 07 artigos. O Quadro 1 mostra os resultados quantitativos dessa busca em pesquisa realizada na data de 20 de dezembro de 2019. 
NARRATIVAS DIGITAIS DE PROFESSORES: UMA REVISÃO SISTEMÁTICA EM TESES E DISSERTAÇÕES BRASILEIRAS
Marcus Alexandre de Pádua Cavalcanti Bastos Eliane Cristina Tenório Cavalcanti Marcelo de Jesus Pereira Shirley Araújo Cabral

Nathan da Costa Cavalcanti Bastos

Quadro 1. Quantidade de trabalhos encontrados no Banco de Teses e Dissertações da CAPES, com a utilização dos descritores "narrativa digital" AND "professor".

\begin{tabular}{|l|l|}
\hline Descritores utilizados na pesquisa & Número de trabalhos \\
\hline Narrativas digitais & 85 \\
\hline Narrativa digital AND Professor & 72 \\
\hline Professor AND Narrativa digital & 07 \\
\hline
\end{tabular}

Fonte: Elaborada pela autora.

Mediante esse resultado panorâmico, optou-se por utilizar os materiais bibliográficos encontrados na busca das palavras-chave associadas “Professor' AND 'Narrativa digital', por serem os descritores que mais se relacionam com o objetivo desta pesquisa".

\section{RESULTADOS E DISCUSSÃO}

Após a aplicação dos critérios e o refinamento da busca a partir da leitura dos artigos pré-selecionados, como descrito na metodologia, foi possível alcançar como resultado 07 trabalhos distribuídos na seguinte ordem: autor(es), título, instituição, tipo de trabalho, ano e programa. O Quadro 02 mostra os resultados quantitativos dessa busca em pesquisa realizada na data de 20 de dezembro de 2019.

Alguns trabalhos não traziam de forma explícita o assunto das narrativas digitais ou algum tema relacionado nos títulos e resumos, o qual se configurou como um problema na pesquisa, sendo, preciso então fazer uma leitura das palavras-chaves, linhas de pesquisa, introdução e até o texto na íntegra para ser possível uma categorização e na tentativa de encontrar a motivação dos pesquisadores para o desenvolvimento dos estudos realizados.

Quadro 2. Trabalhos selecionados para análise na pesquisa

\begin{tabular}{|l|c|l|c|c|c|c|}
\hline No & Autor & \multicolumn{1}{|c|}{ Título } & Instituição & $\begin{array}{c}\text { Tipo de } \\
\text { trabalho }\end{array}$ & Ano & Programa \\
\hline 1 & $\begin{array}{l}\text {. SANTOS, } \\
\text { Vanessa } \\
\text { Aparecida }\end{array}$ & $\begin{array}{l}\text { Tenho um tablet, e } \\
\text { agora? narrativas de } \\
\text { professores de ciências } \\
\text { sobre a inserção da } \\
\text { tecnologia digital na } \\
\text { prática docente (T01) }\end{array}$ & $\begin{array}{c}\text { Universidade } \\
\text { Federal de Itajubá }\end{array}$ & Dissertação & 2016 & $\begin{array}{c}\text { Ensino de } \\
\text { Ciências }\end{array}$ \\
\hline 2 & $\begin{array}{l}\text { CASTELANO, } \\
\text { Karine Lobo }\end{array}$ & $\begin{array}{l}\text { Escrita e autoria escolar: } \\
\text { criação e produção de } \\
\text { narrativas digitais na }\end{array}$ & $\begin{array}{c}\text { Universidade } \\
\text { Estadual do Norte }\end{array}$ & Tese & 2017 & $\begin{array}{c}\text { Cognição e } \\
\text { Linguagem }\end{array}$ \\
\hline
\end{tabular}




\begin{tabular}{|c|c|c|c|c|c|c|}
\hline & & $\begin{array}{l}\text { formação de professores } \\
\text { (T02) }\end{array}$ & $\begin{array}{c}\text { Fluminense Darcy } \\
\text { Ribeiro }\end{array}$ & & & \\
\hline 3 & $\begin{array}{l}\text { CANANI, Carlos } \\
\text { Eduardo }\end{array}$ & $\begin{array}{l}\text { Narrativas Digitais de } \\
\text { Professores: } \\
\text { Perspectivas } \\
\text { Educomunicativas Para } \\
\text { As Práticas Pedagógicas } \\
\text { (T03) }\end{array}$ & $\begin{array}{l}\text { Universidade do } \\
\text { Planalto } \\
\text { Catarinense }\end{array}$ & Dissertação & 2018 & Educação \\
\hline 4 & $\begin{array}{l}\text { CONCEIÇÃO, } \\
\text { Daiane Leal da }\end{array}$ & $\begin{array}{l}\text { Ensino da Matemática: } \\
\text { Potencialidades de Uso } \\
\text { em Concepções e } \\
\text { Práticas Docentes (T04) }\end{array}$ & $\begin{array}{c}\text { Universidade } \\
\text { Federal de Pelotas }\end{array}$ & Dissertação & 2018 & Educação \\
\hline 5 & $\begin{array}{c}\text { TOQUETÃO, } \\
\text { Sandra Cavaletti }\end{array}$ & $\begin{array}{l}\text { Narrativas digitais } \\
\text { multimodais na } \\
\text { formação de professores } \\
\text { da educação infantil } \\
\text { (T05) }\end{array}$ & $\begin{array}{c}\text { Pontifícia } \\
\text { Universidade } \\
\text { Católica de São } \\
\text { Paulo }\end{array}$ & Dissertação & 2018 & Educação \\
\hline 6 & $\begin{array}{l}\text { ARGUELHO, } \\
\text { Miriam Brum }\end{array}$ & $\begin{array}{l}\text { Aprendi fazendo! } \\
\text { Enquanto } \\
\text { aprendia, ensinava: } \\
\text { formação de professores } \\
\text { mediada pelo scratch } \\
\text { (T06) }\end{array}$ & $\begin{array}{c}\text { Universidade } \\
\text { Católica Dom } \\
\text { Bosco }\end{array}$ & Tese & 2018 & Educação \\
\hline 7 & $\begin{array}{l}\text { MADDALENA, } \\
\text { Tania Lucía }\end{array}$ & $\begin{array}{l}\text { Digital Storytelling: } \\
\text { uma experiência de } \\
\text { pesquisa-formação na } \\
\text { cibercultura (T07) }\end{array}$ & $\begin{array}{l}\text { Universidade do } \\
\text { Estado do Rio de } \\
\text { Janeiro }\end{array}$ & Tese & 2018 & Educação \\
\hline
\end{tabular}

Fonte: Elaborado pela autora.

$\mathrm{Na}$ tentativa de responder a questão levantada nesta pesquisa que dizia respeito a como as narrativas digitais vêm sendo utilizadas por professores do ensino básico e superior no país, a busca encontrou 5 (cinco) trabalhos no campo da educação, 1 (um) trabalho na área de Ensino de Ciências e 1 (um) trabalho no campo da Cognição e Linguagem. Essa realidade revela que as pesquisas que tratam das narrativas digitais de professores estão intrinsecamente ligadas à área educacional.

Todos os 07 (sete) trabalhos selecionados possuem a característica de serem empíricos, sendo que 4 (quatro) destes são dissertações e 3 (três) são teses conforme pode ser observado na Tabela 1. 
NARRATIVAS DIGITAIS DE PROFESSORES: UMA REVISÃO SISTEMÁTICA EM TESES E DISSERTAÇÕES BRASILEIRAS
Marcus Alexandre de Pádua Cavalcanti Bastos Eliane Cristina Tenório Cavalcanti Marcelo de Jesus Pereira Shirley Araújo Cabral Nathan da Costa Cavalcanti Bastos

Tabela 1. Distribuição por ano e tipo de material publicado

\begin{tabular}{|l|l|l|l|}
\hline Ano de publicação & \multicolumn{1}{|c|}{ Teses } & Dissertações & Total \\
\hline 2016 & 00 & 01 & 01 \\
\hline 2017 & 01 & 00 & 01 \\
\hline 2018 & 05 & 03 & 02 \\
\hline
\end{tabular}

Fonte: Elaborado pela autora.

Além dos trabalhos trazerem à baila o Professor e Narrativa digital, as produções permeiam diferentes motes como: Ensino de Ciências; Cognição e Linguagem e Educação. É necessário ressaltar que para essa análise seguiremos a ordem dada no Quadro 02 do presente trabalho.

O trabalho T01 teve por objetivo investigar os efeitos do processo de construção de narrativas digitais na percepção de professores de ciências sobre o uso dos tablets na prática docente. O estudo foi de caráter qualitativo cujo percurso metodológico se desenvolveu a partir da confeç̧ão de narrativas digitais que permitiram ao professor rememorar suas experiências relacionadas à tecnologia, em especial ao uso pedagógico do tablet.

Já o estudo 02 apresenta os resultados de uma intervenção realizada com a técnica da narrativa digital (ND), cujo objetivo geral foi investigar se o estímulo de licenciandos a uma relação autoral com a escrita, por meio da produção de NDs, pode influenciar no desempenho da escrita deles e de seus alunos. Para tanto. A metodologia utilizada foi a pesquisa intervenção a fim de compreender a a criação das narrativas digitais. Os resultados dessa experiência demonstraram que a construção de narrativas digitais facilita a relação que os licenciandos e têm com a escrita, uma vez que os sujeitos, ao reconhecerem sua autoria nos diversos usos e combinações expressivas de linguagens, apresentam crescente coesão, coerência e criatividade.

O trabalho T03 buscou analisar as perspectivas educomunicativas que emergem na produção de narrativas digitais por professores do sistema municipal da educação de Lages SC, em relação à utilização das Tecnologias Digitais da Informação e Comunicação (TDIC). Nesse sentido, foram tematizadas as mídias digitais a partir de conceitos que traduzem a 
NARRATIVAS DIGITAIS DE PROFESSORES: UMA REVISÃO SISTEMÁTICA EM TESES E DISSERTAÇÕES BRASILEIRAS
Marcus Alexandre de Pádua Cavalcanti Bastos Eliane Cristina Tenório Cavalcanti Marcelo de Jesus Pereira Shirley Araújo Cabral

Nathan da Costa Cavalcanti Bastos

ressignificação dos comportamentos dos sujeitos nas diferentes esferas da vida cotidiana, com implicações importantes para a educação. A pesquisa desenvolvida foi de caráter qualitativo, com uma abordagem fenomenológica-hermenêutica. Os resultados desta investigação sugerem que as TDIC vêm sendo utilizadas na vida social e nas escolas desses professores e parte deles desenvolve atividades que se aproximam de uma perspectiva educomunicativa.

Seguindo a seqüência foi analisado o estudo T04 que teve como objetivo principal investigar as concepções dos docentes sobre as potencialidades do uso de Aplicativos Educacionais no ensino da Matemática, em um contexto educacional em que o uso dessas tecnologias digitais móveis vem sendo proibido ou restrito ao uso pedagógico. Os sujeitos da pesquisa foram 31 professores de Matemática em atuação na educação básica, da rede pública de ensino de diferentes regiões do Brasil.

O estudo T05 investigou como são produzidas as narrativas digitais multimodais em uma escola de educação infantil, bem como compreender o potencial dessas narrativas como recurso na formação de professores, numa perspectiva crítico-colaborativa. A pesquisa baseou-se na abordagem teórico-metodológica. O contexto da pesquisa foi uma escola pública municipal de São Paulo. Os participantes são uma gestora e dez professores que compõem um grupo de formação da escola em questão. Os dados foram produzidos a partir dos três instrumentos de coleta: questionário semiestruturado sobre registro e documentação pedagógica; os excertos da gravação de áudio das sessões reflexivas e a produção, pelos professores, das narrativas digitais multimodais com recorte das cenas de temas específicos. A análise dos dados foi realizada pelo processo de descrever, informar, confrontar e reconstruir práticas pedagógicas e pela análise multimodal com base nas categorias enunciativas, descritivas e linguísticas. Os resultados indicaram uma desconstrução do senso comum, pelos professores, sobre as produções audiovisuais que permeiam a educação infantil. O processo reflexivo proposto nesta pesquisa sugeriu o desenvolvimento dos participantes por meio de um novo plano de formação em Cadeia Criativa para produzir novos significados às narrativas compartilhadas

O estudo T06 buscou conhecer as concepções dos professores sobre as tecnologias e suas relações com a prática docente. Buscou-se compreender que elementos emergem nesse contexto de formação no sentido de (re)significar o uso das tecnologias - mais 
NARRATIVAS DIGITAIS DE PROFESSORES: UMA REVISÃO SISTEMÁTICA EM TESES E DISSERTAÇÕES BRASILEIRAS
Marcus Alexandre de Pádua Cavalcanti Bastos Eliane Cristina Tenório Cavalcanti Marcelo de Jesus Pereira Shirley Araújo Cabral

Nathan da Costa Cavalcanti Bastos

especificamente do scratch e analisar as implicações da formação com e para as tecnologias, na prática desses professores. O campo empírico da investigação foi um espaço virtual, constituído por um grupo fechado na rede social Facebook. A pesquisa foi de caráter qualitativa, do tipo formação e pretendeu contribuir para a formação continuada de professores para a utilização de tecnologias com base no desenvolvimento profissional e humano dos docentes, por meio da ação reflexiva, na criação e resolução de problemas relacionados às tecnologias no contexto escolar.

Finalizando a seqüência, foi analisado o estudo T07 que abordou a Digital Storytelling (narração digital de histórias) como ato de currículo na formação de professores, tendo como principal objetivo compreender como o conto de histórias por meio digitais pode potencializar as narrativas de si dos professores em formação. A abordagem teórico-metodológica da pesquisa inspirou-se na perspectiva epistemológica da multirreferencialidade $\mathrm{O}$ campo de pesquisa foi realizado na turma da disciplina Tecnologia e Educação do curso de Pedagogia na Universidade do Estado do Rio de Janeiro - UERJ.

\section{CONSIDERAÇÕES FINAIS}

Este trabalho teve como objetivo realizar uma revisão sistemática de teses e dissertações que tratam de narrativas digitais de professores atuantes em escolas de Educação Básica e Educação Superior no país. A análise permitiu observar que o tema foi pouco explorado no cenário brasileiro até 2017. Os resultados apontam que a partir de 2018 houve uma mudança significativa no que se refere ao aumento de pesquisas sobre as narrativas digitais de professores. Entre as áreas mais exploradas, o destaque está para a Educação, em seguida vêm os trabalhos na área de Ensino de Ciências e Cognição e Linguagem.

Os resultados apresentados neste artigo não esgotam todas as possibilidades de análise acerca do tema, tendo em vista que poderiam haver inúmeras outras questões, perfis, bases e suportes que enriqueceriam ainda mais as discussões, todavia, foi possível conhecer algumas tendências de pesquisas. Como toda revisão sistemática, pode-se considerar que o presente estudo vem servir para nortear o desenvolvimento de futuros projetos, indicando novos rumos para futuras investigações. 
NARRATIVAS DIGITAIS DE PROFESSORES: UMA REVISÃO SISTEMÁTICA EM TESES E DISSERTAÇÕES BRASILEIRAS
Marcus Alexandre de Pádua Cavalcanti Bastos Eliane Cristina Tenório Cavalcanti Marcelo de Jesus Pereira Shirley Araújo Cabral Nathan da Costa Cavalcanti Bastos

\section{REFERÊNCIAS}

ALMEIDA, M. E. B.; VALENTE, J. A. Integração currículo e tecnologias e a produção de narrativas digitais. Currículo sem Fronteiras, v. 12, n. 3, p. 57-82, 2012.

ARAÚJO, Cristina Freitas Gonçalves de. TV digital e convergência de mídias: estudo exploratório sobre narrativas interativas. 2010. 129 f. Dissertação (Mestrado em Televisão Digital: Informação e Conhecimento) - Universidade Estadual Paulista Júlio de Mesquita Filho, Bauru, SP, 2010. Disponível em: https://www.faac.unesp.br/Home/PosGraduacao/MestradoeDoutorado/TelevisaoDigital/cristiana-freitas-goncalves-dearaujo.pdf. Acesso em: 2 dez. 2019.

BARTHES, Roland. Introdução à análise estrutural da narrativa. In: BARTHES, Roland et al. Análise estrutural da narrativa. 8. ed. Petrópolis: Vozes, 2013. p. 19-62.

BELL, K.R. Online 3.0-The rise of the gamer educator the potential role of gamification in online education. 2014. 166. (Order No. 3635727) - University of Pennsylvania, Ann Arbor, 2014.

BRUNER, J. Atos de significação. 2a ed.Trad. Sandra Costa. Porto Alegre: Artmed, 2002.

The narrative construction of reality. Critical Inquiry, v.17, p.1-21, 2007.

CLARKE, Robert; ADAM, Andrea. Digital storytelling in Australia: academic perspectives and reflections. Arts and Humanities in Higher Education, v. 11, n. 1-2, p. 157-176, 2012. Disponível em: eric.ed.gov/?id=EJ955497. Acesso em: 12 dez. 2019.

CUNHA, M.I. CONTA-ME AGORA! as narrativas como alternativas pedagógicas na pesquisa e no ensino. Revista da Faculdade de Educação, v. 23 n. 1-2, São Paulo Jan./Dec. 1997.

FENTY, Nicole S.; ANDERSON, Elizabeth. Creating digital narratives: guideline for early childhood educators. Childhood Education, v. 92, n. 1, p. 58-63, 2016. Disponível em: http://eric. ed.gov/?id=EJ1086121. Acesso em: 3 dez. 2019.

FREIRE, Patrícia de Sá. Aumente a qualidade e quantidade de suas publicações científicas: manual para elaboração de projetos e artigos científicos. Curitiba: Crv, 2013.

FREIRE, Paulo. Pedagogia do oprimido. 11. ed. Rio de Janeiro: Paz e Terra, 1987.

GALVÃO, C. Narrativas em Educação. Ciência \& Educação, v. 11, n. 2, p. 327-345, 2005.

LOVELL, Sue; BAKER, Sarah. Digital narratives of youth transition: engaging university students through blended learning. Youth Studies Australia, Austrália, v. 28, n. 4, p. 52-59, 2009. Disponível em: http://eric.ed.gov/?id=EJ867260. Acesso em: 2 dez. 2019. 
NARRATIVAS DIGITAIS DE PROFESSORES: UMA REVISÃO SISTEMÁTICA EM TESES E DISSERTAÇÕES BRASILEIRAS
Marcus Alexandre de Pádua Cavalcanti Bastos Eliane Cristina Tenório Cavalcanti Marcelo de Jesus Pereira Shirley Araújo Cabral

Nathan da Costa Cavalcanti Bastos

MILLER, Caroline Handler. Digital storytelling: A Creator"s Guide to Interactive Entertainment. 3. ed. Burlington, EUA: Focal Press, 2014.

RODRIGUES, A.; ALMEIDA, M. E. B. Narrativas digitais na educação e na formação de professores: uma revisão sistemática de literatura. Cadernos de Educação, v.1, n. 56, p. 107-130, 2017.

RODRIGUES, Alessandra; GONÇALVES, Lina Maria. Narrativas digitais na formação de professores: da memória, do registro e do discurso emergem posturas e experiências. Revista Contexto e Educação, ljuí, v. 29, n. 94, p. 212-237, 2014. Disponível em: https://www.revistas.unijui.edu.

br/index.php/contextoeducacao/article/view/3979/4673. Acesso em: 19 dez. 2019.

POLKINGHORNE de Validity issues in narrative research. Qual Inq. 2007;

ROBIN, Bernard R.; MCNEIL, Sara G. What Educators Should Know about Teaching Digital Storytelling. Digital Education Review, Texas, v. 22, p. 37-51, dez. 2012. Disponível em: eric. ed.gov/?id=EJ996781. Acesso em: 12 dez. 2019.

ROBY, Teshia Young. Opus in the classroom: Striking CoRDS with content-related digital storytelling. Contemporary Issues in Technology and Teacher Education, v. 10, n. 1, p. 133144, 2010. Disponível em: http://eric.ed.gov/?id=EJ904607. Acesso em: 15 dez. 2019.

RODRIGUES, Alessandra. Narrativas digitais, autoria e currículo na formação de professores mediada pelas tecnologias: uma narrativa-tese. 2017. $274 \mathrm{f}$. Tese (Doutorado em Educação: Currículo) - Pontifícia Universidade católica de São Paulo, São Paulo, 2017. Disponível em: <https://tede2.pucsp.br/handle/handle/20196>. Acesso em: 07 dez. 2019.

SANTOS, Vanessa Aparecida Schumann; RODRIGUES Alessandra, REZENDE JUNIOR, Mikael Frank. A construção de narrativas digitais por professores de ciências: análise de uma experiência de formação mediada pelas tecnologias. Revista Brasileira de Ensino de Ciências e Matemática , v. 2, p. 99-118, 2019.

SANTOS, Vanessa Aparecida. "Tenho um Tablet, e agora?": Narrativas de professores de Ciências sobre a inserção da Tecnologia Digital na prática docente. 2016. 97 f. Dissertação (Mestrado Profissional em Ensino de Ciências) - Universidade Federal de Itajubá, Itajubá, 2016.

CARVALHO, G. S. As Histórias Digitais: Narrativas no Século XXI. O Software Movie Maker como Recurso Procedimental para a Construção de Narrações. Dissertação (Mestrado em Educação), Universidade de São Paulo. São Paulo, 2008. Disponível em: http://www.teses.usp.br/teses/disponiveis/48/48134/tde-27082010-104511/publico/ Gracinda_Souza_de_Carvalho.pdf. ACESSO em: 28 out. de 2019. 
NARRATIVAS DIGITAIS DE PROFESSORES: UMA REVISÃO SISTEMÁTICA EM TESES E DISSERTAÇÕES BRASILEIRAS
Marcus Alexandre de Pádua Cavalcanti Bastos Eliane Cristina Tenório Cavalcanti Marcelo de Jesus Pereira Shirley Araújo Cabral Nathan da Costa Cavalcanti Bastos

VAZ, Arnaldo, MENDES, Regina, MAUÉS, Ely. Episódios e narrativas de professores: experiências e perspectivas docentes discutidas a partir de pesquisa sobre conhecimento pedagógico de conteúdo. In: REUNIÃO ANUAL DA ANPED, 24, 2001, Caxambu. Anais... Rio de Janeiro, 2001. p. 1-12.

XIN, Joy F. Digital stories in writing instruction for middle school students with autism. CSCanada: Studies In Literature and Languages, Quebec, v. 9, n. 1, p. 1-10, 2014. Disponível em: http:// www.cscanada.net/index.php/sll/article/view/5180/6211. Acesso em: 9 dez. 2019 